\title{
Use of Melanin for Structural Colors
}

\section{Ali Dhinojwala}

The University of Akron, Ohio, United States

Melanin is a black pigment that is found in many organisms including bacteria, fungi, plants, and animals. This pigment is also responsible for vibrant colors observed in hummingbirds, wild turkeys, and peacocks. The spatial arrangement of melanin in barbules along with keratin creates structural colors that are very iridescent. For example, a large amount of disordered melanin in the barbules of crow produces black color. When these melanin are arranged in a periodic structure results in colors that depend on the size and spacing of melanin granules.

Inspired by this idea, we have recently developed synthetic melanin particles using dopamine as a starting monomer and polymerized melanin particles of various sizes. By using the emulsion-based approach we have self-assembled these melanin particles in clusters to produce structural colors. The spacing between the melanin particles was controlled using a silica shell and by varying the thickness of the silica shell, we can control the color in the visible range.

In my presentation, I will describe the scheme we have used to produce structural colors using melanin and how we have extended this approach to print colors on various materials including paper, plastic, and metals. This simple one-pot process makes it very economical to produce structural colors. In addition, melanin is very stable under UV light and this offers the advantage of producing colors that do not fade with long exposure to sunlight. Interestingly, the refractive indices and absorption coefficient increase with UV radiation and makes these materials more efficient in blocking UV light.

Evolution over a period of around 4 billion years has used chemistry to make materials with very unique properties. At the University of Akron, we have started a Biomimicry Center that brings together faculty members working across many disciplines (biology, physics, chemistry, polymers, and engineering). This collaborative effort has led to the development of new emerging technologies for adhesives, sustainable colors, designing functional structures to better manage heat and light. I will also share some of those experiences in this area in my presentation.

\section{References}

Xiao, M.; Hu, Z.; Wang, Z.; Li, Y.; DiazTormo, A.; LeThomas, N.; Wang, B.; Gianneschi, N. C.; Shawkey, M. D.; Dhinojwala, A., "Bio-Inspired Bright Non-Iridescent Photonic Melanin Supraballs", Sci. Adv. 2017, 3, e1701151. 